\title{
EyeSi - A Simulator for Intra-ocular Surgery
}

\author{
Markus A. Schill ${ }^{1}$, Clemens Wagner ${ }^{1}$, Marc Hennen ${ }^{1}$, Hans-Joachim Bender ${ }^{2}$, \\ and Reinhard Männer ${ }^{1}$ \\ 1 Lehrstuhl für Informatik V \\ Universität Mannheim, B6,26, D-68131 Mannheim, Germany \\ http://www-mp.informatik. uni-mannheim.de \\ 2 Institut für Anästhesiologie und Operative Intensivmedizin \\ Fakultät für klinische Medizin Mannheim der Universität Heidelberg \\ D-68135 Mannheim, Germany \\ markus.schilleti.uni-mannheim.de
}

\begin{abstract}
We present a computer-based medical workstation for the simulation of a vitrectomy that allows training and rehearsal of eye surgeons. The surgeon manipulates two original instruments inside a cardanically suspended mechanical model of the eye. The instrument positions are tracked by CCD cameras and monitored by a PC which then renders the scenery using a computer graphical model of the eye and the instruments. Stereoscopic images are presented to the user through two small LCD displays that are mounted to the system and emulate the stereo microscope used in real operations. The simulator offers the training of intra-ocular navigation as well as first approaches to interaction with pathological tissues using mass-spring and 3D-ChainMail models. All operations (tracking, rendering, collision detection, tissue manipulation) are computed in real-time on a PC.
\end{abstract}

\section{Introduction}

Operating inside the eye is one of the most demanding tasks in microsurgery: the involved structures are extremely sensitive, the field of view is limited and the operation is performed under a microscope, making hand-eye coordination very difficult. In general, two instruments are inserted into the eye. One is a lamp that lights the operation area. The other one is an operative instrument, used to interact with the pathological tissue. There are several operative devices which mainly differ in the way they interact with the tissue. They range from simple picks and cutters to highly sophisticated instruments like the vitrector. In the following we will focus on the vitrector which performs both sucking and cutting. With the vitrector, the vitreous humor, a gelatineous substance filling the eye, is removed and the eye is refilled with a clear liquid. This process is called vitrectomy and is performed in almost all intra-ocular surgeries to either remove an opaque vitreous humor or to obtain free access to the background of the eye.

Figure 1 illustrates a vitrectomy. Typical pathologies which make intraocular surgery necessary include the mentioned opaque vitreous humor, diabetic 
retinopathy and detached retinas. In case of diabetic retinopathy the surgeon has to peel off pathological membranes covering the retina. In this case the vitrector can also be used to remove these membranes. From the simulation point of view the vitrector is one of the most demanding operative instruments.

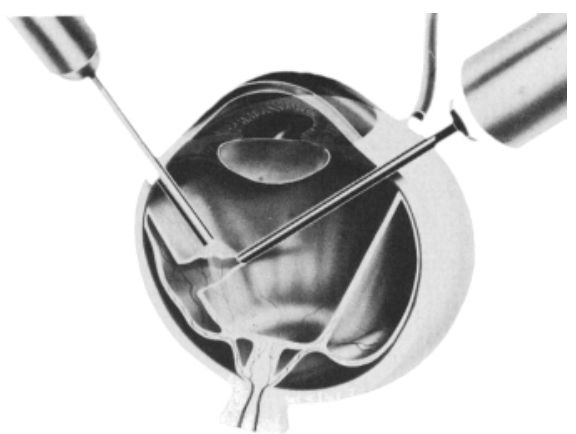

Fig. 1. Illustration of a vitrectomy. The vitrector is inserted from the right, the lamp from the left side. Picture taken from Freyler: Augenheilkunde (1985)

Interacting with pathological tissues in the eye is very difficult. But even the pure navigation of the operative instruments inside the eye is a delicate task. Collisions with the highly damageable retina can be fatal and have to be avoided in any case. The stereo microscope, used in eye surgery, provides three dimensional view, which is a first means of orientation inside the eye. To estimate the distance between a surgical instrument and the retina, the surgeon additionally uses the strong shadow the instrument casts onto the retina with the lamp.

Currently surgeons acquire practical knowledge in intra-ocular surgery entirely by assisting an experienced surgeon. This takes usually two years and bears risks for the patients and the surgeons. The risky, long, and expensive training could be very much improved by using a simulation system that provides the surgeon with a realistic operation environment.

\section{Previous Work on Eye Surgery Simulation}

There are several projects which have dealt with eye surgery simulations in the past $([6,4,1])$. [4] and [6] focus on the simulation of cataract surgery. They either use Finite Element simulations to calculate tissue reaction or previously measured interaction forces. The first could not be accomplished with interactive rates whereas the latter is limited to pre-defined interaction points. In addition [5] describes a current project at the University of Illinois which also aims at the development of a vitrectomy simulator. The project emerged from [1], the 
development of an anatomical eye atlas. Based on this atlas, the simulation provides detailed graphics which also include periphery of the eye like eye muscles and parts of the face. In contrast to [6] and [4,5] uses mass spring models to achieve interactive simulation rates. All mentioned projects use OpenGL surface graphics and expensive graphics workstations for rendering.

From our point of view, the Illinois simulator, as presented in [5], lacks an adequate mechanical setup. An eye model is absolutely necessary to provide realistic instrument handling and a simulation of the eye's movement during surgery. Also, including a high precision tracking of instruments- and eye motion is a prerequisite for a correct simulation: the scale of real motion and simulated feed back must be the same.

\section{The EyeSi Simulator for Intra-ocular Surgery}

Within the EyeSi project a computer-based training workstation for eye surgeons is developed. A prototype allowing the training of membrane peeling is already operational. To reach a high degree of immersion all important aspects of the surgery have been modeled, including the mechanical setup of the surgeon's complete operation environment.

The EyeSi Simulator consists of a model of the operation table, a mechanical eye in which original instruments can be inserted, an optical tracking system for two instruments and the eye motion, an off-the-shelf PC which generates the real-time computer graphics, and stereo glasses that replace the operation microscope.

\section{Mechanical Setup}

A mechanical eye with gimbal suspension was designed and placed on a model of the operation table. Like the real eye in its orbital cavity the mechanical eye provides three rotational degrees of freedom. Springs with appropriate spring constants were used on all three rotational axes to model the back driving forces of the eye muscles. The eye model has two small holes at the positions where the instruments are to be inserted. Real operative instruments are used in the simulation. Figure 2 shows a picture of the eye model with the two instruments inserted.

To model the stereo microscope a pair of active high resolution LCD stereo glasses are used. The arm holding the glasses has two joints and allows the user to individually adjust their position similar to the real microscope. Figure 3 gives an overview of the complete mechanical setup.

\section{Optical Tracking}

During an eye surgery the two instruments are fixed at the insertion points. The tip of each instrument has 3 translational degrees of freedom. In addition the instruments can be rotated. The eye can be tilt in three axes, adding another 3 

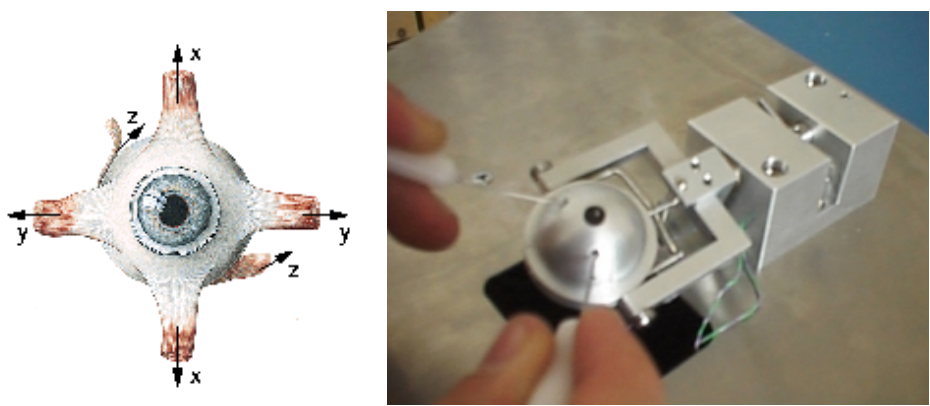

Fig. 2. The real eye in its orbital cavity has three rotational degrees of freedom (left). In the mechanical model, this behavior is modeled by a gimbal suspension and springs which pull teh eye back into its rest position. (right) eye model with two instruments inserted.

degrees of freedom to the system. All these movements must be identified in real-time. We use an optical tracking system for this task.

Optical tracking systems provide high accuracy and work contact free. Unfortunately we did not find a commercial solution that meets our requirements of narrow setup space (the tracking system has to fit into the body of the simulator) and high accuracy in the small volume of interest, essentially the eye volume (approx. $3 \times 3 \times 3 \mathrm{~cm}^{3}$ ). Together with the Fachhochschule Mannheim - Hochschule für Technik und Gestaltung, a solution based on three CCD cameras was developed. Each one is equipped with a digital signal processor (DSP) which can be used to analyze recorded images. The cameras are mounted under the operation table looking from below into the eye model. All instruments and the equator of the eye are marked with small light sources. The DSP in each camera is used to find the projections of the light spots on the CCD sensor and to determine the center of mass of each light spot. These values are then transfered to the PC. As the cameras see the scenery under different angles the stereoscopic back projection can be used with two of the camera images to calculate the positions of the light sources in space. The third camera is automatically activated when an occlusion occurs on one camera. The position of the instruments and the orientation of the eye are then updated accordingly in the computer graphical model.

The equator of the eye model is marked with two light emitting diodes (LEDs) which is sufficient to track its three rotational degrees of freedom. Mounting LEDs to the operative instruments was not feasible because of their small size and possible destruction by mechanical contact during simulation. Instead we abraded the tips of two of the original lamps to uncover the optical fiber inside. The other end of the fiber was connected to a LED. Since the instruments showed sufficient irradiation from their tips and produce good signals on the CCD sensors we used lamps to model all other instruments in the simulator. 

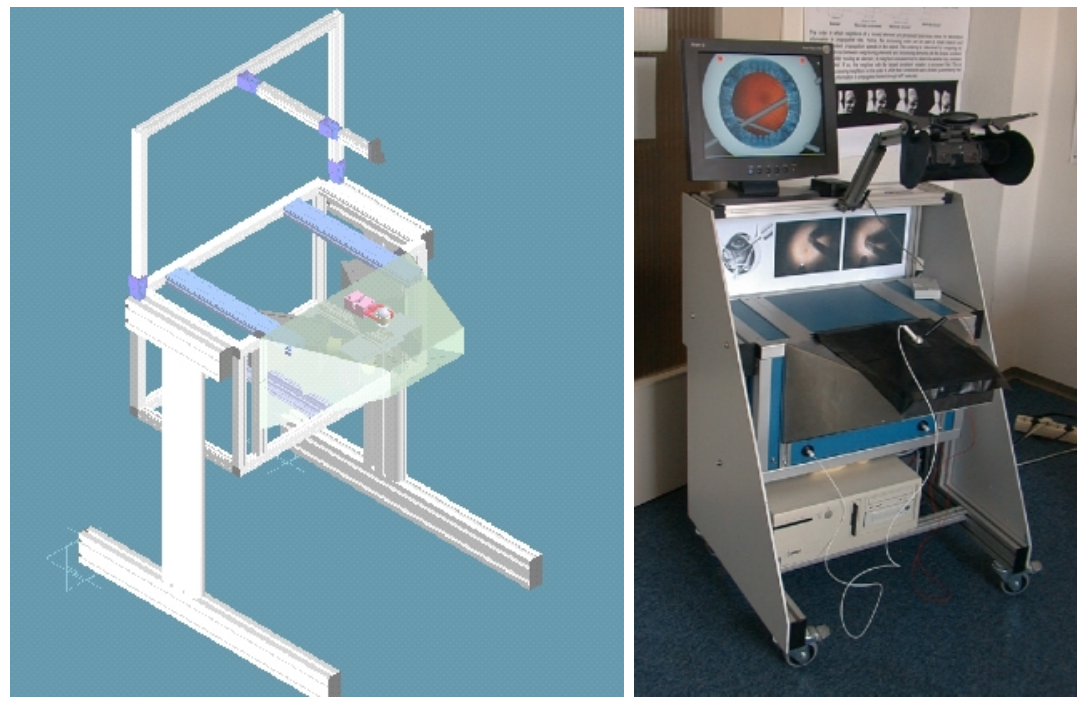

Fig. 3. CAD model of the mechanical setup (left). The current simulator prototype (right).

Our tracking algorithm currently delivers 4 LED positions at $17 \mathrm{~Hz}$. It returns the position of one light spot within $14 \mathrm{~ms}$. We achieve an inplane accuracy of $<0.1 \mathrm{~mm}\left(\approx \frac{30 \mathrm{~mm}}{512 \text { Pixel }}\right)$. The depth accuracy (z-resolution) is about a factor four worse $(\approx 0.3 \mathrm{~mm})$. The spatial resolution of the current tracking system is sufficiently high for the simulated type of retinal surgery. It lies in the same range as the tremour that is observable in a setup like the one used in this kind of surgery. Unfortunately the time resolution of the current tracking system is not high enough for a tremour analysis. (Nyquistś theorem: max. $8.5 \mathrm{~Hz}$ tremour frequency is detectable with the $17 \mathrm{~Hz}$ sampling rate of the tracking system.)

\section{Visualization}

The computer graphical model is based on anatomical data from literature. It is currently rendered using OpenGL surface graphics. The textures used for the iris and the retina are digitized photographies from real eyes. The graphical model includes light effects and shadows. The lamp produces a spot light and the instrument casts a strong shadow on the retina. As mentioned above this shadow is one important means of navigation for the surgeon. The other one is the $3 \mathrm{D}$-view through the stereo microscop. The stereo buffer of OpenGL is used to produce stereo images. Stereo glasses with two high resolution mini LCDs present the calculated pictures to the user. Each LCD has a resolution of $800 \times 600$ and supports truecolor (16.7 million colors).

We achieve interactive framerates on a Pentium $400 \mathrm{MHz}$ PC and a graphics adapter with hardware OpenGL support. Rendering the computer graphical 
model is currently the most time consuming step in the simulation. However, the rendering speed could easily be enhanced by using a graphics adapter with higher triangle throughput.

We are currently upgrading the simulator to include volume visualization. The transparent vitreous humor will be visualized using the volume visualization software developed at the Lehrstuhl für Informatik V, Universität Mannheim [7,3], and provided by Volume Graphics $\mathrm{GmbH}^{1}$.

Up to now simple geometric intersection calculations are used to detect collisions between instruments and eyeball. Due to the spherical shape of the eye ball this can be accomplished very fast. As soon as collisions with more complex shapes have to be considered, e.g. pathological tissues in the eye, collision detection becomes more expensive. In order to accelerate collision detection in these cases we are currently evaluating a new hardware supported approach.

\section{Biomechanics}

Based on 3D-ChainMail [2] an Enhanced ChainMail algorithm for the simulation of inhomogeous materials was developed [8]. This algorithm was successfully tested and will be used in the simulator for the modeling of the vitreous humor. It is currently ported to the EyeSi development platform. The Enhanced ChainMail algorithm has advantages over mass-spring regarding numerical stability. This is particularly true for 3D modeling.

However, since there was an immediate need for membrane simulation, a mass spring model was implemented. It produces good results within the known limitations of mass spring models (see below). The model consists of a triangulated mesh of mass points and springs. The governing equation is

$$
\boldsymbol{F}_{\boldsymbol{i}}=\left(\sum_{j}^{N} \frac{\boldsymbol{x}_{\boldsymbol{j}}-\boldsymbol{x}_{\boldsymbol{i}}}{\left\|\boldsymbol{x}_{\boldsymbol{j}}-\boldsymbol{x}_{\boldsymbol{i}}\right\|}\left(C_{i, j}\left\|\boldsymbol{x}_{\boldsymbol{j}}-\boldsymbol{x}_{\boldsymbol{i}}\right\|-R_{i, j}\right)\right)-D_{i} \dot{\boldsymbol{x}}_{\boldsymbol{i}}
$$

where $\boldsymbol{F}_{\boldsymbol{i}}$ is the force on mass $i, C_{i, j}$ is the spring constant of the spring between $i$ and $j, R_{i, j}$ is the length of this spring and $D_{i}$ the damping of spring $i$.

The biomechanical properties of the model were derived phenomenologically in an iterative process together with an experienced surgeon. With this approach convincing behaviour of soft membranes was achieved. Figure 4 shows an example of membrane peeling off the retina. A membrane tissue with approximately 1,500 mass nodes can be solved within $<10 \mathrm{~ms}$ and runs in the inner simulation loop.

The drawbacks of conventional mass spring systems were experienced when simulating stiffer structures. The system then showed a tendency to numerical instability. Moreover, also in cases were soft membranes were simulated, well conditioned boundary constraints had to be chosen: to avoid a collapse of the triangulated mesh the tissue had to be fixed allong its rim.

\footnotetext{
${ }^{1}$ http://www.volumegraphics.com
} 

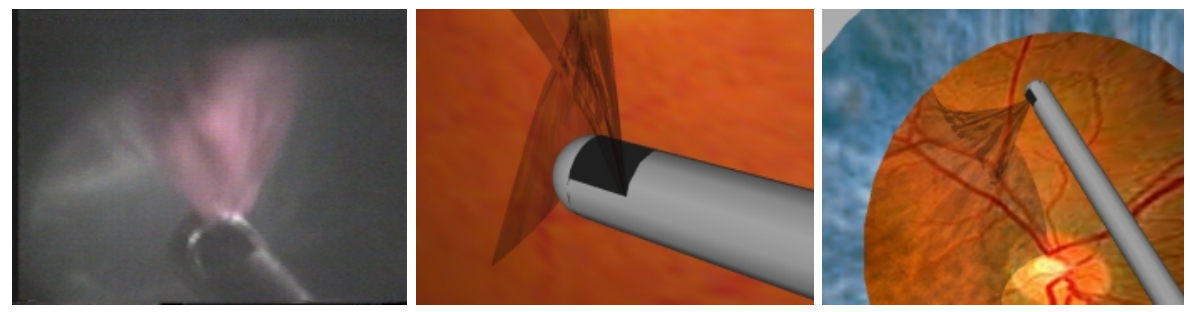

Fig. 4. Video capture (left) and simualtion snapshots (center and right) showing membrane peeling.

\section{Results}

We present a simulator for the training of intra-ocular surgery which incorporates all essential details from the real operation senario. Currently it is possible to exercise the manipulation of instrument inside the narrow space of the eye. Biomechanical models allow interaction with pathological tissues. The actual prototype contains the training of membrane peeling off the retina.

Figure 5 gives a comparison between simulation and real operation. The top row shows screen shots from the simulator, the bottom row video captures from a real surgery. The images illustrate that the simulation is very close to reality: light effects, shadow, eye's background and instruments are modeled conform to the real surgery.

Great importance is also attached to the handling of instruments during the simulation. The mechanical eye (see Figure 2) provides a tactile feedback which is very close to what a surgeon experiences when operating a real eye.

The plattform currently used is off-the-shelf-pc hardware. Depending on the actual simulation task we achieve frame rates between $12 \mathrm{~Hz}$ and $25 \mathrm{~Hz}$. The first rate is for a simulation with membrane in stereo mode where all frames need to be rendered twice, the latter for mono mode. The frame rate breakdown when switching from mono to stereo mode illustrates the bottleneck in OpenGL performance of the current hardware.

If necessary, performance can easily be improved by upgrading the engaged hardware. In particular, future biomechanical modeling might require the change to a multiprocessor system.

We have not yet accomplished clinical evaluation studies, but the prototype will be tested in the Klinikum Mannheim as soon as possible. Nevertheless we received very positive feedback from the eye surgeons who have tried the simulator so far. 

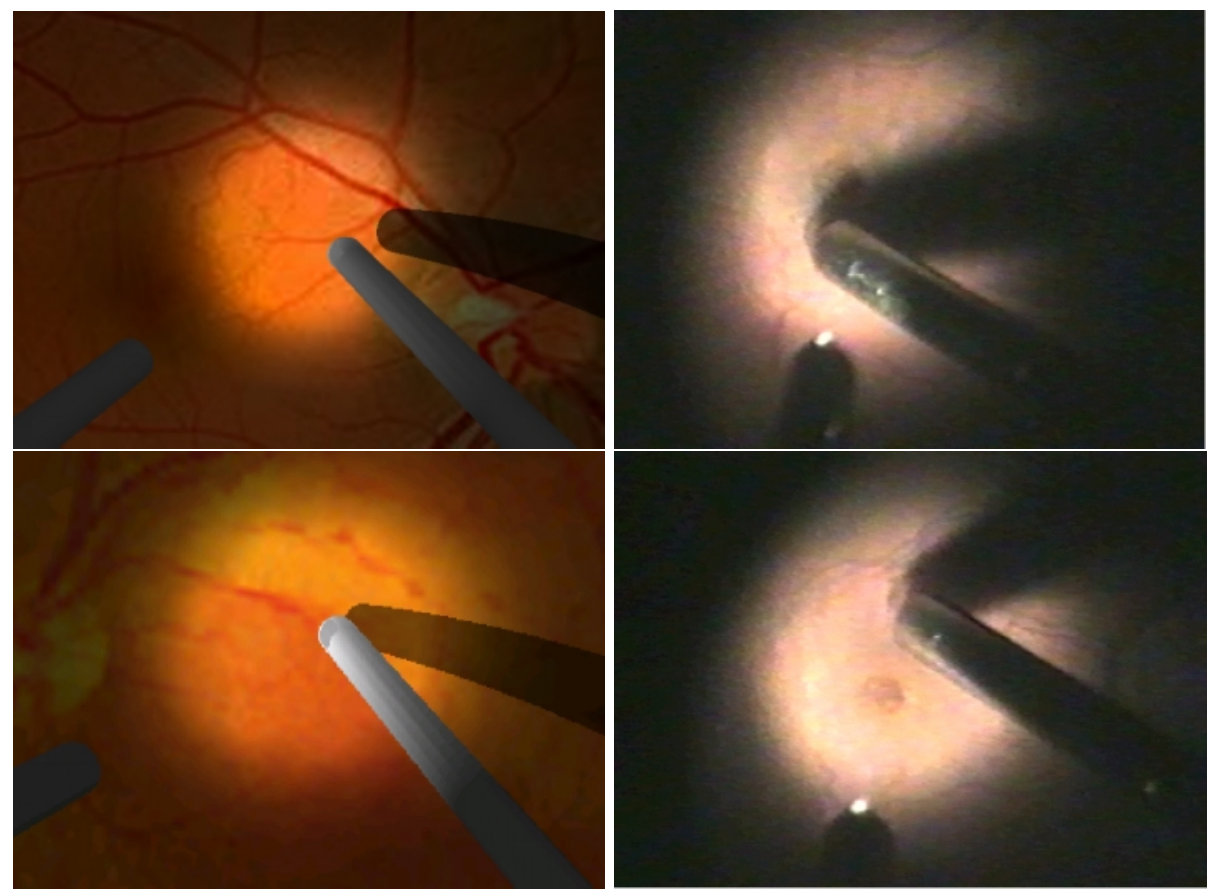

Fig. 5. Comparison: Screenshots from the simulation on the left, video captures from a real surgery on the right.

\section{Discussion}

First estimations claim that by the use of the EyeSi simulator the current period of education (two years) can be cut down by at least three months.

In addition to training the simulator can also be used for the development of new operative instruments and techniques. New instruments can rapidly be prototyped in a simulation. New operation techniques can be developed, tested, demonstrated and taught to colleagues.

Currently an enhanced tracking system is under development which provides about one order of magnitude higher resolution in time and space. This will be obtained by changing the camera type from area to line camera. Volume modeling for biomechanics with the Enhanced ChainMail Algorithm and the usage of volume visualization is work in progress. Up to now the rotation of the operative instruments is not tracked. This will be fixed in the near future.

Future developments will include a training curriculum for surgeons in education. The program will provide a performance analysis informing the trainee about his training success in terms of accuracy and time needed to complete the task. 


\section{Acknowledgement}

The authors would like thank Dr. Fridolin Scheuerle and Gerhart Hospach from EM Gerätebau, Mannheim, for generously supporting the EyeSi project.

Dr. Michael Knorz greatly improved the simulator with his contributions from the eye surgeon's point of view.

Bausch \& Lomb Surgical GmbH, Germany supplied the instruments used in the simulator.

Thanks also to SONY Deutschland GmbH for providing the stereo glasses LDI 100.

\section{References}

1. Biomedical Visualization Laboratory at University of Illinois. Model of the Eye. http://www.bvl.uic.edu/bvl/eye/. 1167

2. S. Gibson. 3D ChainMail: A Fast Algorithm for Deforming Volumetric Objects. In 1997 Symposium on Interactive 3D Graphics, pages 149 - 154, Providence, RI, USA, April 1997. 1171

3. A. Gröpl, T. Günther, J. Hesser, J. Kröll, R. Männer, C. Poliwoda, and C. Reinhart. Interactive Operation Planning and Control with VIRIM. In Proc. Medicine Meets Virtual Reality 4, pages 121-133, San Diego, CA, January 1996. 1171

4. Gordon D. Mallinson Mark A. Sagar, David Bullivant and Peter J. Hunter. A Virtual Environment and Model of the Eye for Surgical Simulation. In SIGGRAPH '94, Anual Conference Series, pages 205-212, July 1994. 1167, 1168

5. Paul F. Neumann, Lewis L. Sadler, and Jon Gieser M.D. Virtual Reality Vitrectomy Simulator. In Alan Colchester William M. Wells and Scott Delp, editors, Medical Image Computing and Computer- Assisted Intervention - MICCAI '98, pages 910 - 917, Cambribge, MA, USA, October 1998. Springer. 1167, 1168

6. Medical College of Georgia \& IMTC at GeorgiaTech. Simulation of a Catheract Surgery. http://www.oip.gatech.edu/MMTLPROJ/eye.html. 1167, 1168

7. Markus Schill, Christof Reinhart, Thomas Günther, Christoph Poliwoda, Jürgen Hesser, Martin Schinkmann, H.-J. Bender, and R. Männer. Biomechanical Simulation of Brain Tissue and Realtime Volume Visualisation. Integrating Biomechanical Simulations into the VIRIM System. In Proceedings of the international Symposium on Computer and Communication Systems for Imageguided Diagnosis and Therapy, Computer Assisted Radiology, pages 283 - 288, Berlin, Germany, June 1997. 1171

8. Markus A. Schill, Sarah F.F. Gibson, H.-J. Bender, and R. Männer. Biomechanical Simulation of the Vitreous Humor in the Eye Using an Enhanced ChainMail Algorithm. In Alan Colchester William M. Wells and Scott Delp, editors, Proc.Medical Image Computing and Computer-Assisted Intervention - MICCAI' 98, pages 679 -687, Cambridge, MA, USA, October 1998. Springer. 1171 